\title{
Ku-Band Low Noise Block Converter (LNB) Sync Application Design Using Android Based Solid Dish
}

\author{
Junaidi Salat $^{1}$, Cut Lilis Setiawati ${ }^{2}$, Zikrul Khalid ${ }^{3}$ \\ ${ }^{1}$ Universitas Jabal Ghafur, Indonesia \\ ${ }^{2,3}$ AMIK Jabal Ghafur, Indonesia \\ junaidisalat@unigha.ac.id,2cutlilis@unigha.ac.id,zikrulkhalid@gmail.com
}

\begin{abstract}
Mobile phone users, especially the Android version of the smartphone, are increasingly spoiling their users. It is undeniable that users do not know their age, even many children have an Android version of the smartphone to play with. Meanwhile, in the satellite tracking world, the satellite tracking is still using $T V$ when tracking the satellite so that it feels difficult. ApplicationLow Noise Block Converter is a video view application from a receiver to an Android smartphone that was built to make it easier for tracking satellites to track one of the satellites that you want to lock. This application also includes satellite tracking information facilities such as tracking guides, frequency updates, and satellite location. ApplicationLow Noise Block Converterbuilt with the Android Studio application using the Java programming language. With the creation of the applicationLow Noise Block Converter seas a new alternative to replace $T V$ as a satellite tracking device or monitoring tool.
\end{abstract}

Keywords

lownoise block converte;

dish solid, android

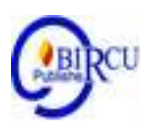

\section{Introduction}

In current technological developments, parabolic antennas with all the equipment have become one of the hardware devices that can be classified into luxury items because the price is quite expensive image quality. This is what makes parabolic antennas widely used, apart from that the price is quite expensive to make parabolic antennas a symbol of prosperity for those who have them. Broadly speaking, the use of a satellite dish is to capture TV broadcasts from satellites in the form of downlink signals transmitted by satellites. The channel that is ready to be channeled through a satellite dish is a channel that already occupies the satellite transponders.

So far, many of the downlink signal receivers emitted by satellites use standard satellite dishes sold in the market, so users have to buy them from sellers at quite expensive prices. And while tracking satellite or looking for satellite signals many residents use TV as a tool to see the satellite signals they get, therefore residents must bring TV when tracking the satellite.

There are also many types of LNBs. Each LNB can only be used for a single frequency band such as S-Band, C-Band and $\mathrm{Ku}$-Band, each frequency requires a different resonator.

In an lnb there is what is called polarity, there is horizontal polarity and vertical polarity. Horizontal polarity requires 18 volts dc power from the receiver, and vertical polarity requires 13 volts dc power supply. If there is one polarity that is off, for example the $\operatorname{lnb}$ is to pick up the palapa satellite signal but the vertical polarity is off, then we cannot catch broadcasts such as Mnc, Matrix tv, Jtv and other channels that are in one polarity with vertical. In a satellite dish the function of the lnb is very important, if our $\ln b$ 
is damaged we will not be able to get a signal from the satellite, which will be interpreted by the receiver and in the end we will not be able to watch the television broadcast on the parabola.

In an lnb there is what is called polarity, there is horizontal polarity and vertical polarity. Horizontal polarity requires 18 volts dc power from the receiver, and vertical polarity requires 13 volts dc power supply. If there is one polarity that is off, for example the lnb is to pick up the palapa satellite signal but the vertical polarity is off, then we cannot catch broadcasts such as Mnc, Matrix tv, Jtv and other channels that are in one polarity with vertical. In a satellite dish the function of the lnb is very important, if our lnb is damaged we will not be able to get a signal from the satellite, which will be interpreted by the receiver and in the end we will not be able to watch the television broadcast on the parabola.

Each LNB can only be used for a single frequency band, because the $\mathrm{S}, \mathrm{C}$ and $\mathrm{Ku}$ bands each require a different resonator cavity. There are also types for linear and circular signals, differentiated by the placement of the internal dipole. The power supply for electronic switches is also of interest. Power is provided by the receiver and supplied via a coaxial cable. So the coax cable not only transmits the signal received from the antenna to the receiver, but also requires operating power from the receiver to the LNB (along with additional control signals).

Switch Features When switching channels a transponder has one or two different polarizations (horizontal or vertical and circular left or right). So the receiver has to tell the LNB the polarization of the given signal, so that the appropriate dipole can be activated. The power supply voltage is as follows: $14 \mathrm{~V}$ activates vertical polarization, while $18 \mathrm{~V}$ activates horizontal polarization. Although DiSEqC has been developed as a powerful control tool with more than 256 commands, it is still not used to shift polarization.

The universal LNB has a second switch mode for the extended $\mathrm{Ku}$ band. Since the satellite receiver frequency range is not wide enough, the frequency range has to be separated into two frequency ranges. Switching between these two ranges is controlled by the $22 \mathrm{kHz}$ signal which the receiver also sends to the LNB when selecting a particular channel. This $22 \mathrm{kHz}$ signal is also used for the carrier frequency for DiSEqC control commands in more complex system configurations. This DiSEqC command can control multiswitches and antenna motors.

\section{Research Methods}

Some of the research methods used by the author are as follows:

1. Interview

Interviewing is the process of obtaining information by conducting direct questions and answers with related parties.

2. Literature review

By studying the material that has been obtained related to the thesis topic through media such as books, articles, writings on internet sites, and other information media.

3. Study of literature

Literature study is a method carried out by utilizing available literature, such as utilizing internet facilities, namely by visiting websites that are related to the problems faced by the author. As well as collecting references to available books.

4. Programming and Implementation

Making applications is done with Java programming language, this system support application in the form of android studio. 


\section{Evaluation}

The evaluation stage is carried out to determine the errors found and collected for analysis as a basis for further application development.

\section{Discussion}

\subsection{Types of LNB}

\section{a. LNB S-Band}

This LNB captures satellite signals with an input frequency of 2,520 $\mathrm{MHz}$ to 2,670 $\mathrm{MHz}$ and an output frequency of $950 \mathrm{MHz}$ to $1,100 \mathrm{MHz}$ with a local oscillator of 2,620 MHz. With a low enough input frequency for a satellite signal, which is 2,520 - 2,670 $\mathrm{MHz}$, of course, the signal will not be affected by weather disturbances such as cloudy and rainy weather. However, with low frequencies, of course, there is also a risk of intervention from other frequencies. In Asia, there is only one satellite that uses this frequency, namely the SES 7 Satellite which is located at the $108^{\circ} \mathrm{E}$ position which is used for paid or subscription parabolic transmissions.

\section{b. Lnb C-Band}

This Lnb captures satellite signals with an input of 3,400 MHz to 4,200 MHz and an output of $950 \mathrm{MHz}$ to $1,750 \mathrm{MHz}$ with a Local Oscillator of 5,150 MHz. The input frequency of 3,400-4,200 MHz tends to be safer from other frequency interventions and is still quite resistant to cloudy and rainy weather disturbances. The satellite that transmits the c-band frequency is very widely used by FTA (Free To Air) tv stations or free tv or nonpaid TV, and there are several pay TVs that use this frequency. The downside of this frequency is that the size of the parabolas must be large.

\section{c. Ku-Band Lnb}

$\mathrm{Ku}-\mathrm{Band} \mathrm{Lnb}$ captures satellite signals with an input of $10,700 \mathrm{MHz}$ to $12,750 \mathrm{MHz}$ and an output of $950 \mathrm{MHz}$ to $2,150 \mathrm{MHz}$ with a double Local Oscillator, namely with a frequency of 9,750 MHz and 10,600 MHz. This Lnb has the highest frequency in its class. In addition, the frequency coverage is wide enough to accommodate many transponders. With high frequency, it is very effective against other frequency interventions, but is vulnerable to being disturbed by cloudy and rainy weather. $\mathrm{Ku}-\mathrm{Band}$ frequencies are mostly used for pay tv and only a few are used for FTA tv.

\subsection{Digital Parabolic Receiver}

According to Cai Wardana (2017) Parabolic Receiver is a device used to receive parabolic signals which will later be applied to television without a satellite dish receiver it will not be possible to broadcast a television program, Parabolic receivers have various types and brands according to their respective advantages.

In the 1990s, the use of receivers for satellite dishes that were originally analog based was starting to be replaced by digital receivers. Of course, the reception process for digital receivers is of a better quality than that of analog receivers. It's just that, the digital receiver in 1996 still had MPEG2 quality. However, along with the development of technology in the satellite dish, especially in the receiver technology used, in the 2000s digital receivers already supported MPEG4. Of course, the technology carried by the MPEG4 receiver is much higher than that of the MPEG2 digital receiver. It's just that, in the 2000s, units of MPEG4 receivers were still quite limited and hard to find. 
2013 can be said to be one of the years when the development of MPEG4 receivers was so fast. This is motivated because the MPEG4 receiver already supports a number of features, for example, being able to record television broadcasts, being able to open randomized broadcasts using Biss Key, cccam features, multimedia facilities, facilities for opening random Tanberg which is quite famous for the difficulty level in opening scrambles, facilities In addition, a number of MPEG4 receivers also have an Androidbased and Linux-based operating system embedded in it.

\subsection{Easy CAP}

According to Iyens Bsa (2017) EasyCAP is a connector tool that functions to connect Audio Video Out (RCA) from TV, Parabolic Receiver, DVD Player, Play Station, Camera/Handycam, CCTV. The important thing is the Audio-Video Out which will be connected to a USB computer or laptop or even an Android cellphone with the help of OTG (On The Go), so that it can be recorded and even edited. This tool is in the form of a USB connector that just plugs into a USB computer or laptop and Android cellphone. Very practical because the shape is very compact, so we can immediately record video and audio without the help of a soundcard or other hardware.

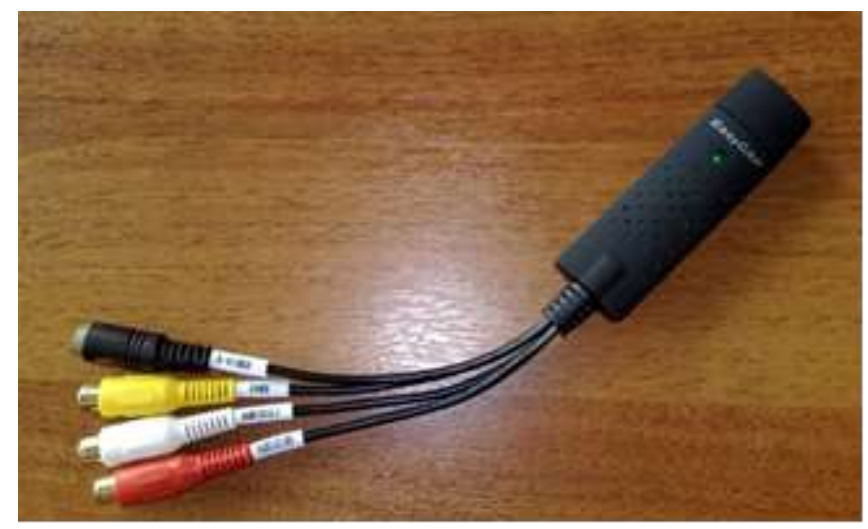

Figure 1. Easy CAP

\subsection{Parabolic Antenna}

According to Aloha (2018), a parabolic antenna is an antenna that has high coverage and usually this parabolic antenna is used in radio, TV communication and also for radiolocation or (RADAR), in the UHF and SHF sections of the electromagnetic wave spectrum. The relatively short wavelengths of electromagnetic energy (radio) at these frequencies can make the size used for a parabolic antenna still within a reasonable size in terms of the high performance response desired to either receive or transmit a signal. The parabolic antenna that we know is shaped like a dish, well it can be seen in front of a house that uses a parabolic antenna, all of them are the same in the form of a round dish, some are small and large.

\section{Types of Parabolic Antennas}

There are several types / types of antennas on the market, including a very common type of dish antenna Solid, Net (Mess):

\section{a. Solid Parabolic Antenna (Dish Solid)}

The SOLID type parabolic antenna made of an alloy plate with alumenium as a reflector is actually divided into two, namely: 
1. The dish is oval or offset focus with a small enough diameter between $45 \mathrm{~cm}$ to $100 \mathrm{~cm}$. This is what we often encounter use for Pay TV such as Indivision, Transvision, KVision, Orange TV, Astro, Aora, Big TV and many more (although some are not for pay tv such as Ninmedia and TV Desa Group).

2. A round dish or prime focus which looks like most satellite dishes sold in the market. The sizes range from 4 to 12 feeds, but most of what is sold and used is only 6 feeds.

\section{b. Mesh Parabolic Antenna (Net)}

Mess type parabolic antenna is a net with small holes as its reflector. The sizes also vary, from 6 feeds, 7.5 feeds, 9 feeds, 12 feeds to 16 feeds, but what we often find and sell is 9 feeds and 12 feeds, although other sizes also use them. The dishes we often know and commonly know are the Paramount and Paragon brand antenna nets.

The advantage of a net antenna is that it is not easy to rust because it is made of aluminum and is not heavy (light) compared to a solid prime focus antenna.

\subsection{Coaxial Cable}

Coaxial cable also known as BNC (Bayonet Naur Connector) or coaxial cable is often used for tv antenna cables and is often used on LAN networks. This cable is usually widely used to transmit high frequency signals from $300 \mathrm{kHz}$ and above. Because of its ability to transmit high frequencies, the transmission system using coaxial cables has a fairly large channel capacity.

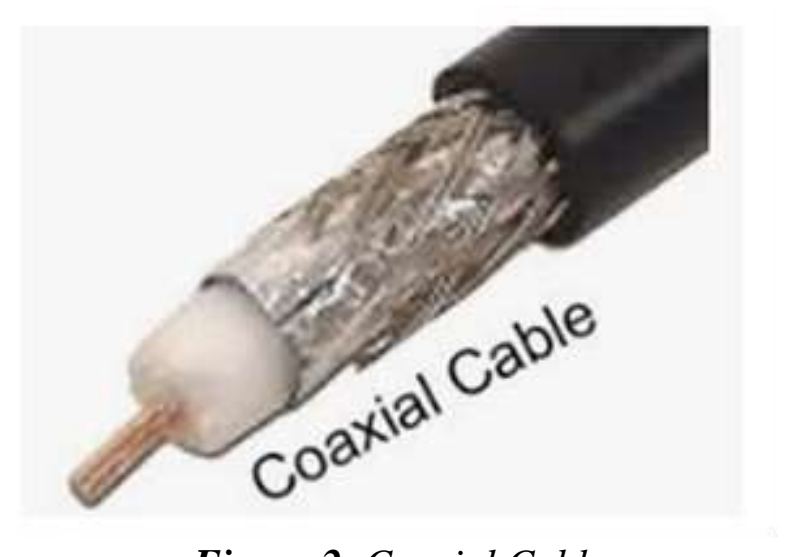

Figure 2. Coaxial Cable

According to Kuwat Kris (2017), a coaxial cable is a network cable wrapped in soft metal. Network installation using this cable is relatively easier than using a UTP cable, but the access speed on the cable is a little slower, so most people don't want to use it. Coaxial cables are more efficient to use for network systems with less capacity because of their access speed which does not allow them to be used on networks with large capacities.

The parts of the coaxial cable:

1. Conductor, this section is the core of the cable that functions as data traffic in the network

2. Grounding is a filamentous cable twisted across and around an inner insulator. This part of the cable serves to anticipate the influence of unwanted electrical frequency interference.

3. The inner insulator is part of the cable that serves to protect the conductor.

The outer isolator is the part of the cable that is located on the outer shell which functions as a cable protector as a whole. 


\subsection{RCA Connector}

According to Isty (2017), the RCA cable is a cable that is usually used to send analog signals from audio or video equipment to other equipment. RCA cables tend to be large in size and each cable is separated by color because it has different uses. The yellow cable is usually used for visuals as a carrier for analog video signals, while the red and white cables are for audio, red cables are for the right channel analog audio signal and the white one is for the left audio channel.

Called the RCA cable or Radio Corporation of America is one of the cables that has an important role to connect audio and video (visual) equipment. These cables are widely used in Indonesia and the ones that are often seen are usually connected from DVD to TV. However, there are also CCTV cables that apply this cable as a connection to cameras and DVRs.

This RCA type connector is probably the one we see the most. This connector is commonly used in cables carrying audio and video (analog) signals. We can see it on a vcd or dvd player or video game when connected to the tv.

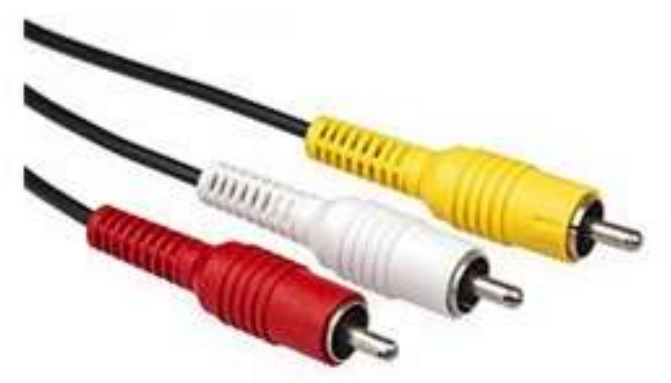

Figure 3. RCA Connector

\subsection{Android}

Android is an Operating System (OS) or operating system which is very popular these days. It is undeniable that this operating system has taken a lot of attention from the world community and the Indonesian people, of course. Gadget and cell phone companies are competing to make devices using the Android operating system. Therefore, it is surprising that almost every day we see new gadgets on the market that use the operating system made by Google.

\section{a. Android Software Development Kit (SDK)}

Android is a software subset for mobile phones that includes the operating system, middleware and key applications released by Google. Currently, the Android SDK (Software Development Kit) is provided as a tool and API to start developing applications on the Android platform using the Java programming language.

\section{b. Android Version 1.1}

On March 9, 2009, the Google company released Android version 1.1. This version is equipped with an aesthetic update to the application, namely an alarm clock, voice search, sending messages with Gmail, and email notifications (Hermawan, 2010).

\section{c. Android Version 1.5 (Cupcake)}

Android Version 1.5 (Cupcake) There is an update in the operating system, including the addition of several features in this version, namely the ability to record and watch videos in camera mode, Bluetooth A2DP support, the ability to automatically connect to 
on-screen animated Bluetooth headsets, and an on-screen keyboard that is can be customized and released in mid-May 2009 (Hermawan, 2010).

\section{d. Android Version 1.6 (Donut)}

Android version 1.6 (Donut) was released in September 2009 and differs from previous versions of Android which features a better search process, battery usage indicator and VPN applet control. Other features are galleries that allow users to have photos to be deleted, integrated cameras, camcorders and galleries, CDMA / EVDO, VPN, Gestures, and text-to-speech engines, dial contact capabilities, provision of WVGA resolution (Hermawan, 2010) .

\section{e. Android Version 2.0 / 2.1 (Éclair)}

Android version 2.0 / 2.1 (Eclair) launched in December 2009 has several changes, namely hardware optimization, changes to the User Interface (UI) with a new browser and HTML support, a new contact list, improved Google Maps 3.1.2, flash support for cameras 3.2 MP, digital zoom, and Bluetooth 2.1 (Hermawan, 2010).

\section{f. Android Version 2.2 (Froyo)}

In May 2010, the Android phone version 2.2 (Froyo) was launched again, changes to version 2.2 include optimization of speed, memory and overall operating system performance, support for installing applications on external memory, support for Adope Flash 10.1 and USB Tethering and Wi-Fi functions. FI (Hermawan, 2010).

\section{g. Android Version 2.3 (GingerBread)}

December 1, 2010 Google re-launched the latest version, Android version 2.3. in this version there is an increase in power management, control through applications, the use of multiple cameras, increased performance and the addition of sensors such as a gyroscope (Hermawan, 2010).

\section{h. Android Version 3.0 / 3.1 (Honeycomb)}

This version is different from previous versions, this version is specifically designed for Tablet PCs so that it has a different User Interface (UI) and supports larger screen sizes. In addition, this version allows the use of multiple processors and hardware acceleration for graphics. The first version of the SDK was launched in February 2011, the next version that will appear, namely the Ice Cream version in the fourth quarter of 2011 (Hermawan, 2010).

\section{i. Android 4.0 (Ice Cream Sandwich)}

The peak of Android maturity is when in this version, which Ice Cream Sandwich was released in October 2011 ago. And this operating system starts working on any kind of smartphone. Apart from adding interesting features, Ice Cream Sandwich was also the Android version most liked at that time. In fact, Android Ice Cream Sandwich is also equipped with extra multitasking features and more notifications.

\section{j. Android 4.1.2 (Jelly Bean)}

Jelly Bean was released on July 9, 2012 through the Google I / O conference. This version is one of the versions of Android that often gets updates on useful and interesting features, some of which are improving screen rotation, such as Support for $4 \mathrm{~K}$ video resolution, Support for writing Hebrew and Arabic letters from right to left, and improved performance, security systems and many others. 


\section{k. Android 4.4 (Kitkat)}

This version of Android is currently used by the majority of Indonesians. Kitkat is the version of Android which was released in 2013. in this version, Android gets a lot of feature updates. For example, there is a Screen recording feature, to record activities that occur on your smartphone screen, New Translucent system UI, improved notification access, System-wide settings for closed captioning, performance improvements and many others.

\section{Android 5.0 (Lollipop)}

Released in 2014, this Android offers more additional features to enhance existing features. And the Nexus 6 is one of the very first phones to taste this version of Android. In addition, Google is also further refining the performance of Android Lollipop itself.

\section{m. Android 6.0 (Marshmallow)}

Android version 6.0 is one of the Android operating systems released in 2015, which brings many updates. One of them is USB Type-C support. Not only that, Android version 6 also provides fingerprint authentication facilities and increased battery power.

\section{n. Android 7.0 (Nougat)}

Android Nougat version 7.0 was released in August 2016 which further improves the performance of the previous Android version. In addition, Android Nougat also gets many new features, including being able to multitask, improving the Doze feature that was released in previous versions of Android.

And here are some of the newest features found in Nougat.

1. Support Multi window

2. Can directly reply to messages from the notification window or menu

3. New notification panel and quick settings display

4. Enhanced Doze mode (Doze Mode 2.0)

5. Menu among system settings

\section{Android 8.0 (Oreo)}

The Android Oreo version was released in August 2017. Of course this Android version is the final version for now. Several features were also launched by Google as the manager. The features include:

1. Android $\mathrm{O}$ focuses more on speed and efficiency

2. 2X faster Boot up speed

3. Picture in picture mode is more flexible than Android $\mathrm{N}$

4. Applications running in the background are more tightened to save battery

5. Longer battery life

6. Updated emojis and more

\section{p. Android Studio}

According to Davidk (2017) Android Studio is the official Integrated Development Environment (IDE) for Android application development, based on IntelliJ IDEA. Android is turning into a platform that is so fast in innovating. This is inseparable from the main development behind it, namely Google. Google was tired of acquiring Android and then building a platform. The android platform consists of a Linux-based Operating System, a GUI (Graphic User Interface), a web browser and downloadable End-User Applications and also the developers can freely work and create the best and open applications for use 
by various devices. IDE (Integrated Development Environment) is a computer program that has several facilities needed in software development.

An IDE, or freely translated as an Integrated Development Environment, has at least the following facilities:

1. Editor is a facility to write the source code of the software.

2. The compiler is a facility to check the syntax of the source code then convert it into binaries according to machine language.

3. Linker is a facility to unify binary data from which some source code is generated by the compiler so that the binary data becomes one unit and becomes a computer program that is ready to be executed.

4. Debuger, which is a facility to test the running of the program, to look for bugs or errors in the program.

To some extent modern IDEs can help provide suggestions that speed up writing. When writing code, the IDE can also point out areas where it is clear that it contains errors or doubts.

\section{q. Project Structure}

Each project in Android Studio contains one or more modules with source code files and resource files. Module types include:

1. Android application module

2. Library Module

3. Google App Engine Module

All build files are visible at the top under Gradle Scripts and each app module contains the following folders:

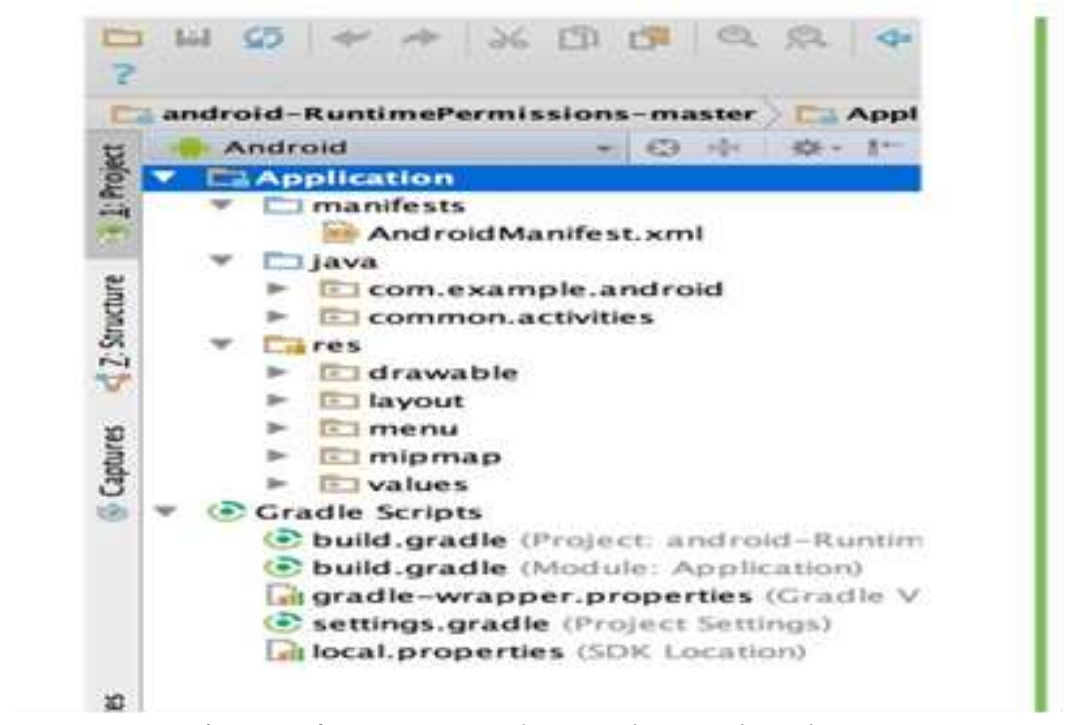

Figure 4. Project Files in the Android View

1. manifests: Contains the AndroidManifest.xml file.

2. java: Contains Java source code files, including JUnit test code.

3. res: Contains all non-code resources, such as XML layouts, UI strings, and bitmap images. 


\section{r. User Interface}

The Android Studio main window consists of several logical fields identified in the following figure:

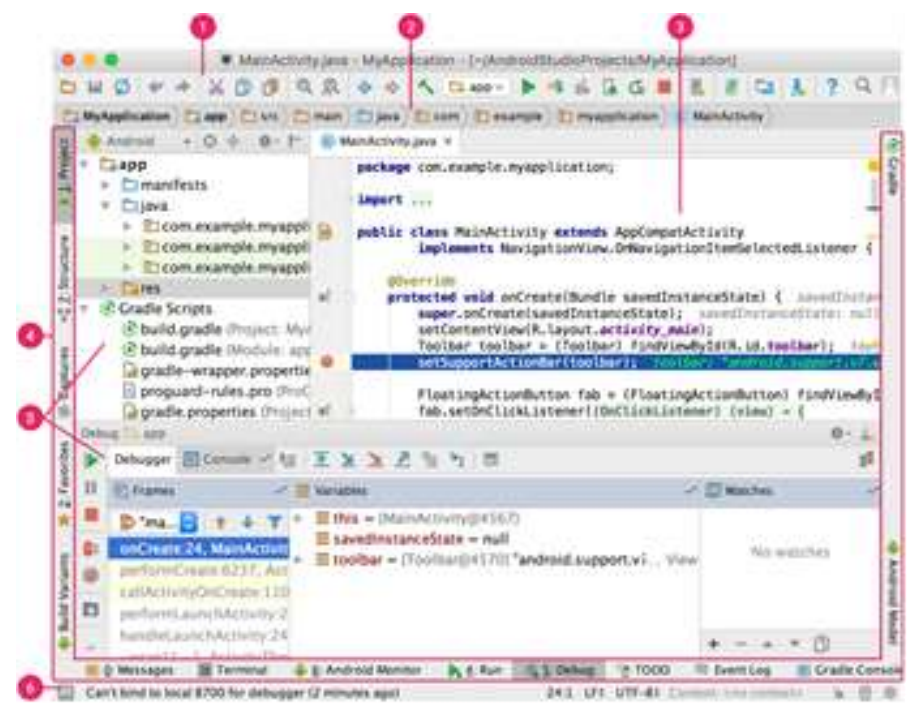

Figure 5. Android Studio Main Window

1. Toolbars allow you to perform various types of actions, including running applications and launching Android tools.

2. The navigation bar helps you navigate between projects and open files for editing. This bar provides a more compact view of the structure in the Project window.

3. The editor window is where you create and modify code. Depending on the current file type, the editor may change. For example, when viewing a layout file, the editor displays the Layout Editor.

4. The tool window bar appears outside the IDE window and contains buttons that let you expand or collapse individual tool windows.

5. The tool window gives you access to specific tasks such as project management, tracing, version control, and more. You can expand as well as collapse.

6. The status bar displays the status of your project and the IDE itself, as well as any warnings or messages.

\section{S. Tools Window}

Rather than using a preset perspective, Android Studio follows your context and automatically pops up relevant tool windows as you work. By default, the most frequently used tools are pinned to the tool window bar at the edge of the application window.

1. To expand or collapse a tool window, click the tool name in the tool window bar. You can also drag, pin, attach, and drop the tool window.

2. To return to the current default tool window layout, click Window $>$ Restore Default Layout or adjust your default layout by clicking Window> Store Current Layout as Default.

3. To show or hide the tool window bar, click the window icon in the lower-left corner of the Android Studio window.

4. To find a specific tool window, hover over the window icon and select that tool window from the menu. 


\section{t. Gradle Build System}

Android Studio uses Gradle as the basis of the build system, with Android-specific capabilities provided by the Android Plugin for Gradle. This system can be run as an integrated tool from the Android Studio menu and independently from the command line. You can use the build system features to do the following:

1. Customize, configure, and extend the build process.

2. Create multiple APKs for your Android app, with different features using the same project and modules. Reuse code and resources across source sets.

By applying Gradle's flexibility, you can achieve all of this without changing your app's core source files. The Android Studio version file is named build.gradle. This file is plain text that uses Groovy open in new to configure the version with elements provided by the Android plugin for Gradle. Each project has a top-level build file for the entire project and a separate module-level build file for each module. When you import the current project, Android Studio automatically generates the required version files.

\section{u. Profiling and Debug Tools}

Android Studio helps you debug and improve the performance of your code, including inline debugging and performance analysis tools.

\section{v. Inline Debug}

Use inline debugging to step up your code in the debugger view with inline verification of reference values, expressions, and variables. Inline debug information includes:

1. Inline variable value

2. Referring object that refers to the selected object

3. The method's return value

4. Lambda and operator expressions

5. Tooltip value

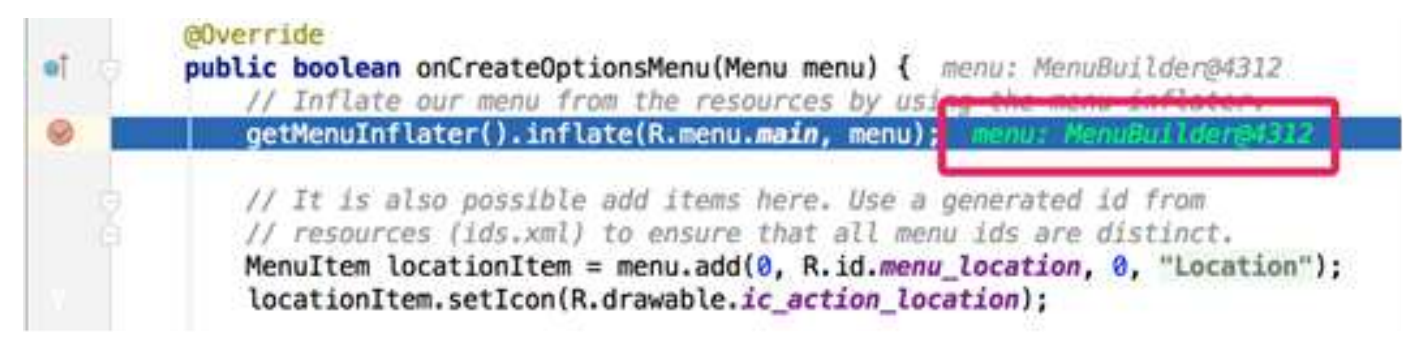

Figure 6. Inline Variable Value

To enable inline debugging, in the Debug window, click Settings and select the checkbox for Show Values Inline.

\section{1) Performance Monitoring}

Android Studio provides performance monitoring so you can more easily track your app's memory and CPU usage, find unallocated objects, find memory leaks, optimize graphics performance, and analyze network requests. With the app running on the device or emulator, open the Android Monitor tool window and click the Monitors tab. 


\section{2) Heap Dump}

When you monitor memory usage in Android Studio, you can simultaneously initiate trash collection and dump the Java heap into a heap snapshot in an Android-specific HPROF binary format file. The HPROF viewer displays classes, instances of each class, and a reference tree to help you track memory usage and find memory leaks.

\section{3) Allocation Tracker}

Android Studio allows you to track memory allocation while monitoring memory usage. Track memory allocation allows you to monitor where objects are allocated when you perform certain actions. Knowing this allocation allows you to optimize application performance and memory usage by adjusting the calling method associated with the action.

\section{4) Access Data Files}

The Android SDK tools, such as Systrace, logcat, and Traceview, generate debugging and performance data for detailed app analysis. To see the availability of the resulting power file, open the Captures tool window. In the list of generated files, doubleclick the file to view the data. Right-click the .hprof file to convert it to the standard hprof file format.

\section{5) Code Check}

Whenever you compile your program, Android Studio will automatically run configured Lint and the IDE Check for open in new helps you easily identify and fix problems with the structural quality of your code. The Lint tool checks your Android project source files for possible bugs and optimization fixes for accuracy, security, performance, usability, accessibility and internationalization.

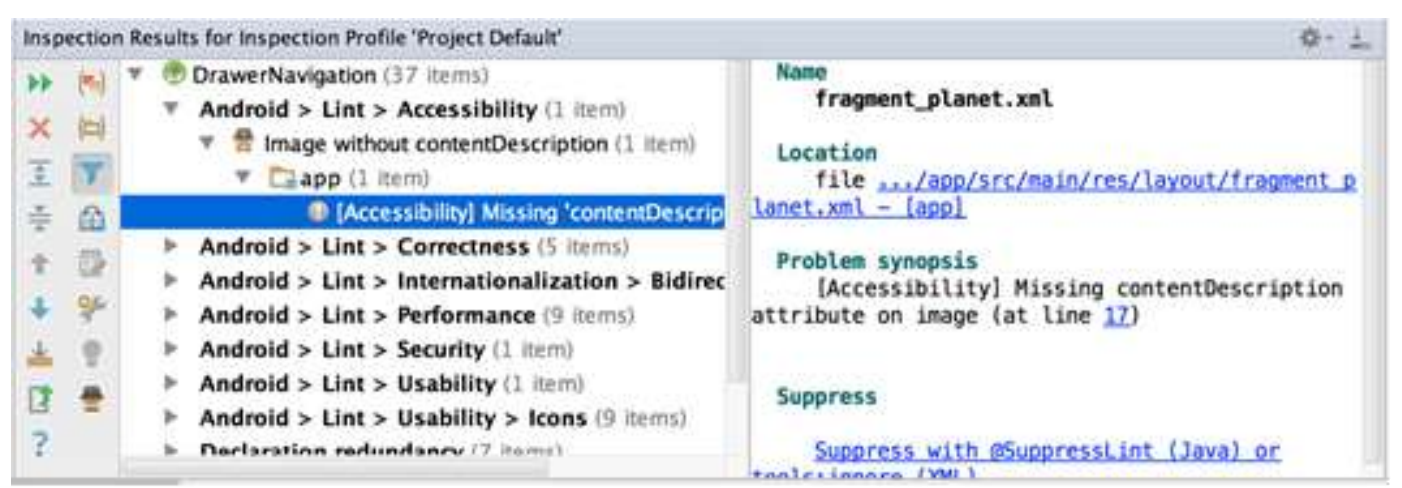

Figure 7. Results of a Lint Check in Android Studio

\section{6) Annotations in Android Studio}

Android Studio supports annotations for variables, parameters, and return values to help you catch bugs, such as null pointer exceptions and resource type conflicts. The Android SDK Manager packages libraries in the Android Support Repository for use with Android Studio. Android Studio will validate configured annotations during code inspection.

\section{7) Log messages}

When building and running your app with Android Studio, you can view device logs and output messages and adb (logcat) by clicking Android Monitor at the bottom of the window. If you want to debug your app with Android Device Monitor, you can open 
Device Monitoring by clicking Tools> Android> Android Device Monitor. In Device Monitor you can find a complete set of DDMS tools for identifying applications, controlling device behavior, and so on. Also included is the Hierarchy Viewer tool to help optimize your layout.

\section{8) Android Debug Bridge (ADB)}

Serves as a versatile command tool allowing you to communicate with an emulator instance or a connected Android device. This facilitates a variety of device actions, such as installing and debugging applications, and provides Unix shell access that you can use to run various commands on the emulator or connected device. It is a client-server program that includes three components:

1. Client, who sends orders. The client runs on your development machine. You can request clients from the command-line terminal by issuing the adb command.

2. Daemons, which execute commands on the device. The daemon runs as a background process on each emulator or device instance.

3. The server, which manages the communication between the client and the daemon. The server runs as a background process on your development machine.

\section{9) Enable ADB Debugging on Your Device}

To use ADB with a device connected via USB, you must enable USB debugging in the device system settings, under Developer options.

On Android 4.2 and up, the Developer options screen is hidden by default. To make it visible, go to Settings> About phone and tap Build number seven times. Return to the previous screen to find Developer options at the bottom. On some devices, the Developer options screen name or location may be different.

You can now connect your device via USB. You can verify that your device is connected by running adb devices from the android_sdk / platform-tools / directory. If connected, you'll see the device name listed as "device".

\subsection{Java}

Java is a high-level, object-oriented programming language and a Java program is composed of parts called classes. Classes consist of methods that do work and return information after doing their job. Java programmers take advantage of the collection of classes in the Java class library, which is called the Java Application Programming Interface (API). These classes are organized into groups called packages. The Java API has provided sufficient functionality for creating sophisticated applets and applications. So there are two things that must be learned in Java, namely learning the Java language and how to use classes in the Java API. Class is the only way of declaring the executable part of the program, there is no other way. In Java the javac program is used to compile Java source code files into bytecode classes. The source code file has the extension * .java. The javac compiler generates class bytecode files with the extension * .class. The interpreter is the main module of the Java system that is used by Java applications and runs Java bytecode programs.

\section{Java Code Structure in Android Studio}

Following are some lines of java code in Android Studio that are automatically generated when you create an activity. 


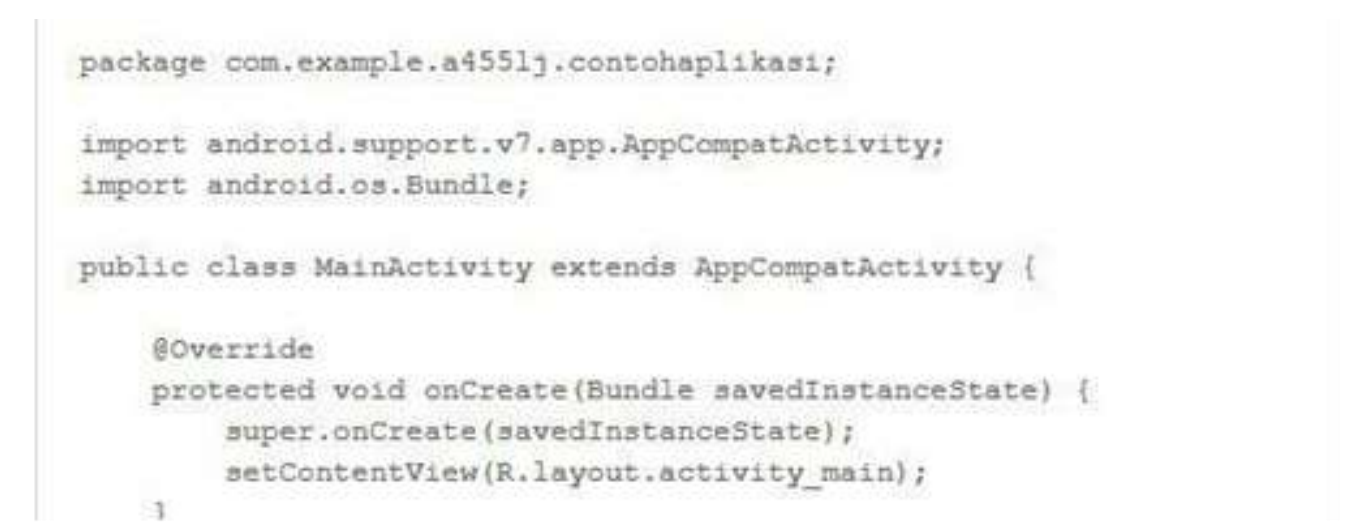

Figure 8. Java Code Structure in Android Studio

\subsection{Github}

According to Candra Adi Putra (2014) Github is a hosting software for open source projects that uses the Git control revision Tool System. So Git is a tool for revising code, while github is the web hosting. Easily Github is Webhosting for software projects such as Google code or sourceforge net. Github is a popular shared web service for software development projects that use a Git version controller system or a hosting service for open source projects that use the Git control revision Tool System. In simple terms, Github is a social network for developers in developing a project. This github site provides social networking functionality and social networking graphics to show how the developers are working on their repository version.

Github also operates other services: a pastebin-style site called Gist which provides a wiki for each repository and an editable web page via a Git repository, a slide hosting service called Speaker Deck, and a web analysis platform called Gauges. As of January 2010, Github is operated under the name Github, Inc. The software that runs Github is written in Ruby on Rails and Erlang by the Github developers.

\subsection{Satellite Tracking}

According to Najwa (2016) Satellite tracking is positioning the dish or parabolic dish towards the satellite that was shot to get the satellite signal. To get the maximum signal from the target satellite, this dish must be pointing exactly right at the satellite. slightly off the mark, so the results are not optimal. might not even get the signal at all. In general, a tool for tracking satellites is called a satellite finder. This tool will help the tracker (the person who is tracking) if the dish that is being tracked leads to a target satellite. after the dish leads to the satellite, we just need to maximize the signal. here is a very important point. The angle of declenation, elevation and azimuth must be precise to maximize the signal received.

\section{Flow Chart}

Flowchart is a chart with certain symbols that describe the sequence of processes in detail and the relationship between a process ith another process in a program. 
Table 1. Flowchart

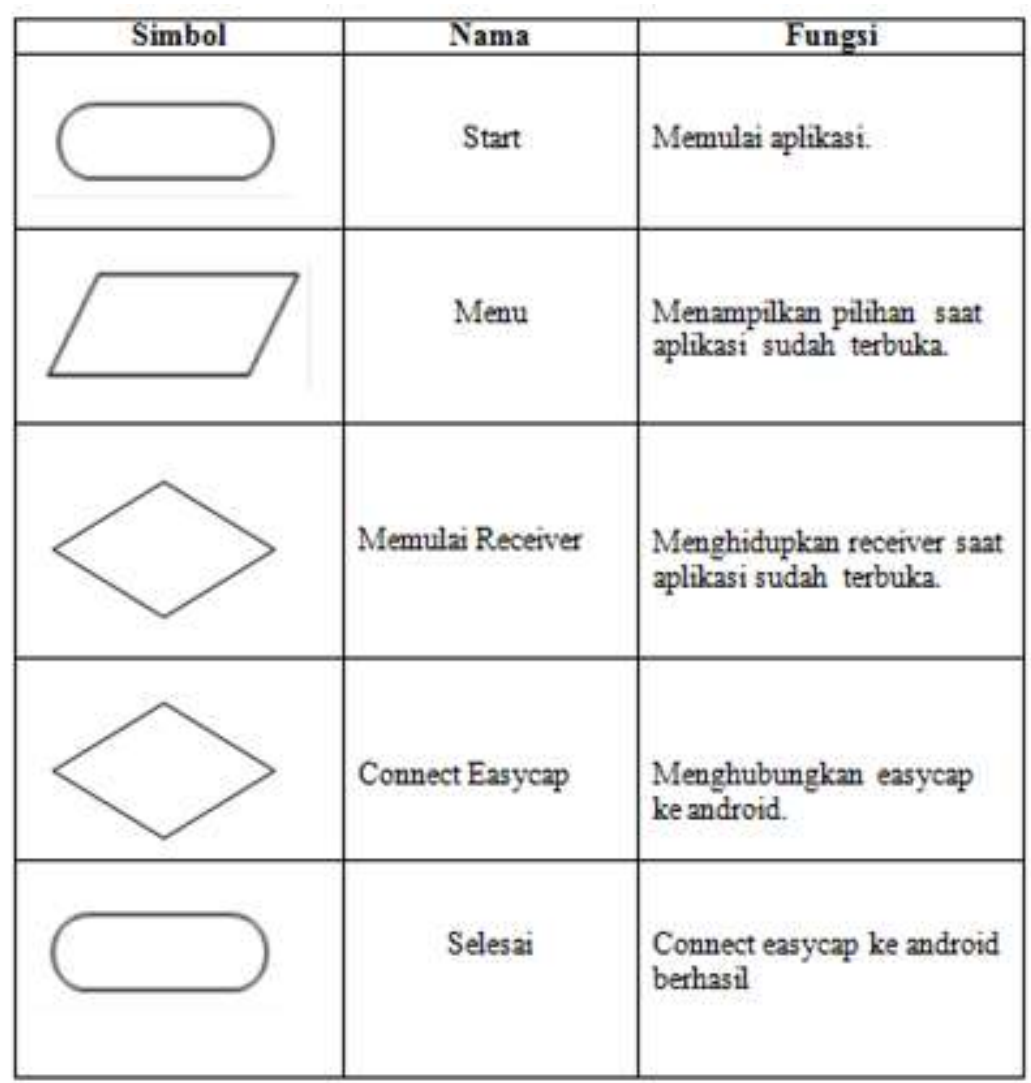

\section{Conclusion}

Based on the results of the design and implementation that has been done, and based on the formulation of the existing problems, several conclusions can be drawn including the following:

1. Making a Low Noise Block Converter application for android smartphones can be made with Android Studio using the java language.

2. Low Noise Block Converter application as an application to facilitate tracking satellites and can run well on android OS version 7.0 nougat.

3. With this application as a new alternative in tracking satellite.

4. Android smartphones can be used optimally by using the Low Noise Block Converter application for satellite tracking.

\section{References}

Aloha, 2018, "understanding parabolic antenna" accessed on November 16, 2018, fromhttps://adalobang.blogspot.com/2018/02/pengentuk-antena-parabola.html.

Bayu Nur Huda, Making and Comparative Analysis of the Performance of a Bolic Griddle and a Can Antenna in Capturing Wifi Signals, Muhammadiyah University of Surakarta, Surakarta, 2014.

Cai Wardana, 2017, "types and types of satellite dish receiver models" accessed on November 16, 2018, from http: // computer-informatics. blogspot.com/2017/05/typeand-model-receiver-parabola.html. 
Candra Adi Putra, 2014, "introduction to github for beginners" accessed on November 16, 2018, from https://www.candra.web.id/pengetakan-github-untuk-pemula/.

Davidk, 2017, "Get to know what android studio is" accessed on November 16, 2018, from https://teknologimodern.com/mengenal-apa-itu-android-studio/.

Much Aziz Muslim, Utilization of a Wok for Wifi Antenna, Journal of Information Technology DINAMIK, Stikubank University Semarang. Volume XIII, No.2, July 2008.

Masputz, 2015, “digital satellite dish receiver" accessed on 16 November 2018, from http://www.masputz.com/2015/06/perb Difference-receiver-digital-parabola.html.

Najwa, 2016, "tools for tracking satellite" accessed on 16 November 2018, from https://najwatehnik.blogspot.com/2016/11/alat-untuk-tracking-satelit.html

Ichsyan Nafik, Yuniarto, Design of Wajan Bolic Antenna with a diameter of 46 centimeters at $1900 \mathrm{Mhz}$ Frequency to strengthen WCDMA signal reception, Journal of Gema Teknologi, Diponegoro University, Semarang, Vol 17 No.1 April 2012.

Iyens Bsa, 2017 "easycap" accessed on 16 November 2018 from https://forsatdoumbozo.blogspot.com/2017/01/nonton-tv-di-komputer-dan-hpdengan_21.html.

Isty, 2017, "getting to know the RCA cable" accessed on 16 November 2018, from https://www.digna.co.id/blog/mengenal-kabel-rca/.

Junaidi Yusuf Arifin, 2013, "the meaning of coaxial cable" accessed on November 16, 2018, from http://denaydeni.blogspot.com/2013/01/pengentuk-kabel-coaxial.html.

Kuwat Kris, 2017, "definition and function of coaxial cable" accessed on November 16, 2018, from http://kuwatkrishd.blogspot.com/2017/02/pengentuk-dan-fungsi-kabelcoaxial-dan.html

Teamplant Rize, 2013, "easycap capture usb video audio adapter" accessed on 16 November 2018, from https://teamplant.blogspot.com/2013/04/easycap-capture-usbvideo-audio-adapter.html.

Tommi Putra, 2014, "getting to know the types of connection cables" in access on November 16, 2018, from https://tommiputra.blogspot.com/2014/05/mengenal-jeniskabel-koneksi.html.

Yolanda, 2018, "getting to know RCA cables" at access on November 16, 2018, from https://www.audioengine.co.id/mengenal-kabel-rca/. 